\title{
Vertigo by Breast Cancer Metastasis 33 Years after Treatment
}

\author{
Atsunobu Tsunoda ${ }^{1}$, Yoji Tanaka ${ }^{2}$, Takanobu Sato ${ }^{3}$, Susumu Kirimura ${ }^{4}$ and Ken Kitamura ${ }^{1}$
}

\begin{abstract}
A 76-year-old woman complained of vertigo for two years. She manifested left deafness, loss of caloric response, and right-beaten nystagmus. An imaging study revealed a tumorous lesion located from the clivus to the left temporal bone with inner ear destruction. A tumor biopsy was performed endonasally and the patient was diagnosed with adenocarcinoma mimicking breast cancer. She had undergone surgery for breast cancer 33 years previously, and the current biopsy specimen showed identical pathology. Breast cancer may metastasize to the skull base; however, metastasis 33 years after surgery is very rare.
\end{abstract}

Key words: breast cancer, hearing loss, skull base, vertigo

(Intern Med 55: 311-313, 2016)

(DOI: 10.2169/internalmedicine.55.5412)

\section{Introduction}

Vertigo is a commonly encountered symptom in the outpatient clinic. Various otologic and non-otologic diseases cause vertigo (1-5). Neurological examinations and imaging studies are important diagnostic interventions; however, careful history taking is also critical for an accurate diagnosis. We herein report a case of breast cancer metastasis to the skull base presenting as vertigo 33 years after the initial therapy.

\section{Case Report}

A 76-year-old woman complained of dizziness and vertigo spells for two years. She also noticed progressive hearing loss in her left ear. She was otherwise healthy, except for a history of right breast cancer. She had undergone mastectomy 33 years previously with no recurrence noted. An initial examination revealed left middle ear effusion. Rightbeating nystagmus, loss of left caloric response, and mixed deafness in the left ear were also noted. No other neurological deficit was found. A nasopharyngeal endoscopic examination showed swelling on the anterior wall of the right sphenoid sinus (Fig. 1A). Blood testing was unremarkable except for hemoglobin A1c (JDS) levels of 6.6\% (normal range: 4.3-5.8). Several tumor markers, such as CA15-3: $182 \mathrm{U} / \mathrm{mL}$ (normal range: <25.0), CA125: $50.7 \mathrm{U} / \mathrm{mL}$ (normal range: $<35)$, carcinoembryonic antigen $(\mathrm{CEA}): 14.1 \mathrm{ng} /$ $\mathrm{mL}$ (normal range: <5.0) and CA72-4: $5.1 \mathrm{U} / \mathrm{mL}$ (normal range: 4.0 ), were also elevated.

On CT and MRI studies, a lesion was demonstrated in the clivus and sphenoid sinus to the left temporal bone with inner ear destruction (Fig. 1B). Despite the high levels of several tumor markers, an 18-fluorodeoxyglucose positron emission tomography (18F-FDG PET)-CT scan revealed no abnormal uptake except for the skull base lesion (Fig. 1C). According to these findings, a primary skull base lesion, such as chordoma, was suspected.

The patient underwent a tumor biopsy under nasal endoscopy. The tumor was granulous and fibrous. On frozen section, the tumor was diagnosed to be adenocarcinoma, which was confirmed by subsequent histological examinations. On immunostaining histology, the tumor was positive for estrogen and progesterone receptors (ER, PgR), gross cystic disease fluid protein (GCDFP) 15, and mammaglobin, which strongly suggested metastasis of breast cancer. The histological section of the primary lesion was obtained from the

\footnotetext{
${ }^{1}$ Department of Otolaryngology, Tokyo Medical and Dental University, Japan, ${ }^{2}$ Department of Neurosurgery, Tokyo Medical and Dental University, Japan, ${ }^{3}$ Department of Breast Surgery, Saiseikai Central Hospital, Japan and ${ }^{4}$ Department of Pathology, Tokyo Medical and Dental University, Japan

Received for publication March 23, 2015; Accepted for publication May 11, 2015

Correspondence to Dr. Atsunobu Tsunoda, atsunoda@mac.com
} 

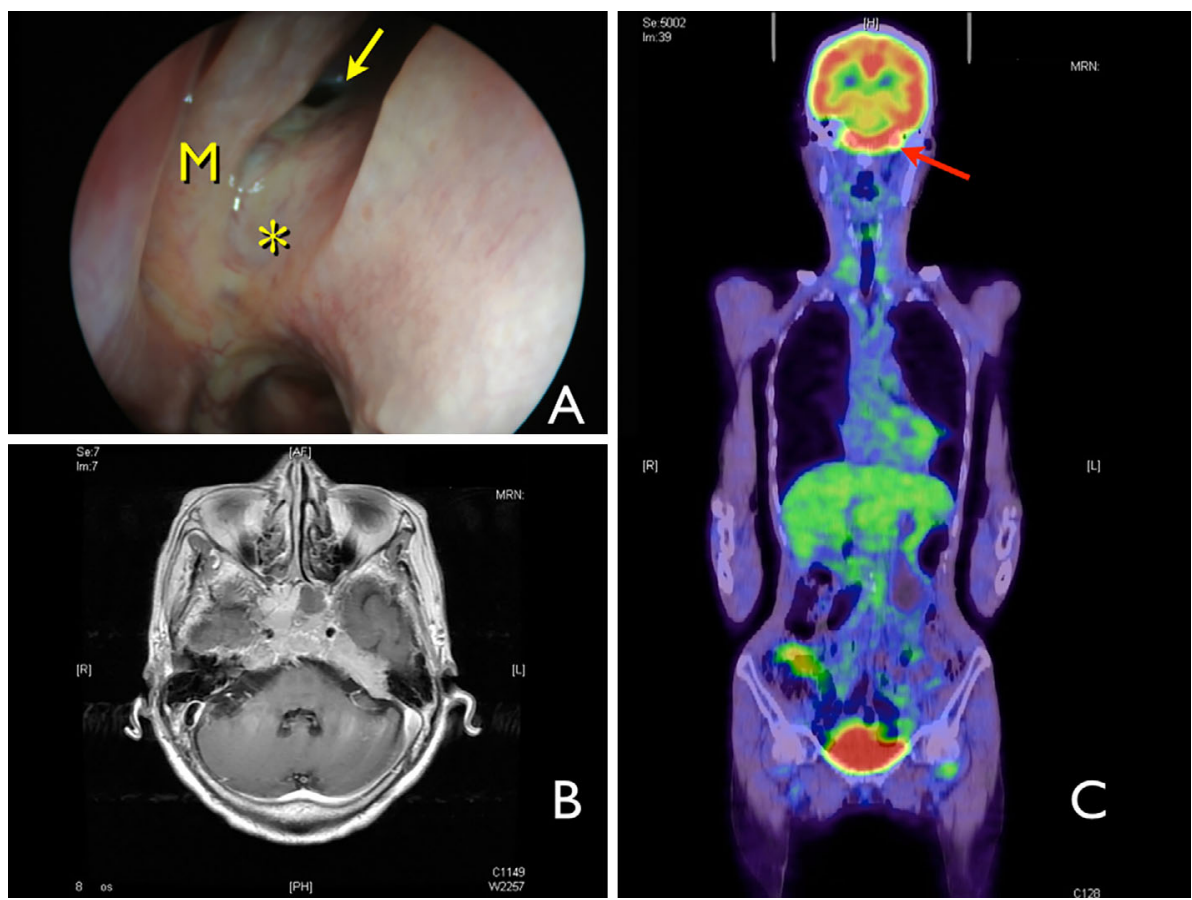

Figure 1. An endoscopic image of the right nasal cavity (A). The anterior wall of the sphenoid sinus shows bulging (*), and the middle turbinate $(\mathrm{M})$ is shifted laterally. The arrow indicates the orifice of the sphenoid sinus. On a T1-weighted MRI image, the large skull base lesion shows a high signal intensity (B). 18F-FDG PET-CT shows a high uptake in the skull base lesion (arrow); however, no other metastasis is noted $(C)$.

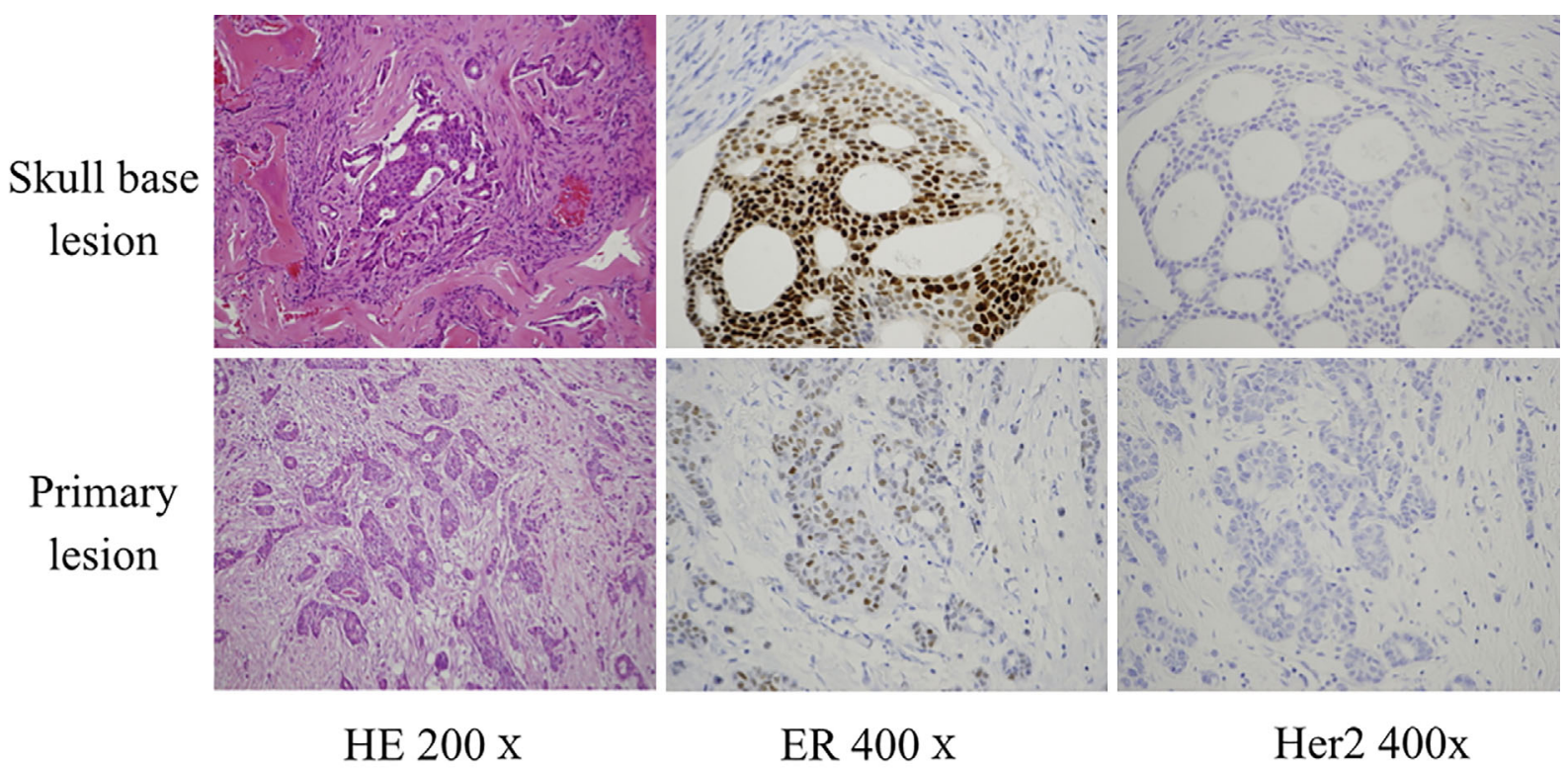

Figure 2. A histological section of the skull base and primary lesions. Both pathologies mimicked and showed histology similar to the previous breast cancer. The primary lesion had similar immunostaining histology namely, positivity for ER, but negativity for HER2.

hospital where she underwent mastectomy. Both pathologic findings mimicked and showed the same histology as that of her previous breast cancer. The primary lesion had similar immunostaining histology, namely, positivity for ER and PgR but negativity for HER2 (Fig. 2). The absence of cancer on the contralateral breast and the findings of $18 \mathrm{~F}-\mathrm{FDG}$ PET-CT led to the diagnosis of the skull base lesion as me- tastasis of breast cancer treated 33 years previously. This metastatic lesion invaded the left inner ear and led to the patient's symptoms. She then underwent radiotherapy of 44 Gy followed by oral tamoxifen therapy. The vertigo spells disappeared and dizziness subsided. The patient has remained free of the disease for two years. 


\section{Discussion}

Vertigo is a very common complaint which is encountered among clinicians, and both peripheral and central vertigo are encountered. Peripheral vertigo, such as benign paroxysmal positional vertigo, is commonly encountered. However, certain vertigo can be caused by very rare conditions, and such conditions may be very difficult to diagnose accurately. Therefore, various diagnostic interventions, such as careful history taking, otological and neurological examinations, and imaging studies, are required for an adequate diagnosis $(1,3-5)$.

Metastasis to the skull base particularly affects patients with breast cancer followed by those with lung and prostate cancers $(6,7)$. Although rare, there are various reports on metastasis of carcinoma, and five common skull base metastatic sites include the orbit, parasellar area, middle fossa, jugular foramen, and occipital condyle with accompanying symptoms. Clinical features due to the progressive ipsilateral involvement of the cranial nerves include headache, ophthalmoplegia, facial numbness, and hoarseness (7-10). Nasal bleeding has also been reported. These symptoms are commonly seen in the clinic; thus, physicians must pay careful attention to the possible presence of skull base metastasis in patients with a previous history of the aforementioned carcinomas. However, breast and prostate cancers sometimes show very late metastasis with no recurrence in the primary site (11-13). The present case showed ER (+) and HER2 (-) on the histological examination and was diagnosed as Luminal A type on both the primary and metastatic sites. Generally, breast cancer is divided into 4 molecular subtypes, and this classification may be useful in planning treatment. The four major molecular subtypes include Luminal A, Luminal B, Triple negative/basal-like, and HER2 type. Among them, Luminal A type shows a good prognosis, however, it is known to show late recurrence (14). A recent study indicated that the bone marrow delivers dormancy signals in exosomes to metastatic cancer cells (15). Such a mechanism potentially occurred in this rare episode.

In such situations, the physician, as well as the patient, rarely notes the history of previously treated cancer. In the present case, the occurrence of metastasis 33 years after surgery was extremely rare, and the possibility of such a late metastasis would not normally cross the mind of a physician. If this lesion did not invade the inner ear, then the patient would not have experienced dizziness and the metastasis would have been discovered much later. Breast cancer may show metastasis in a very late period after initial treatment, such as in the present case, thus the clinician must pay careful attention to a previous history of breast cancer.
The authors state that they have no Conflict of Interest (COI).

\section{Acknowledgement}

The authors are deeply indebted to the staff members of the Cancer Institute Hospital (Tokyo) for providing histological sections obtained over 33 years ago.

\section{References}

1. Covelli E, Attanasio G, Cagnoni L, Viccaro M, Filipo R. Pneumolabyrinth after intratympanic steroid injection in patient with prosthesis of the stapes: a case report. Am J Otolaryngol 34: 759-761, 2013.

2. Martínez-Quintana E, Rodríguez-Gonzílez F. Vestibulopathy after a modified Blalock-Taussig procedure in a cyanotic congenital heart disease patient. Am J Otolaryngol 34: 248-251, 2013.

3. Chou CI, Lin HC, Wu KC, Shu MT. Brainstem hemorrhage presented as audiovestibular syndromes. Am J Otolaryngol 33: 356357, 2012.

4. Kojima H, Tanaka Y, Mori E, Uchimizu H, Moriyama H. Penetrating vestibular injury due to a twig entering via the external auditory meatus. Am J Otolaryngol 27: 418-421, 2006.

5. Tsunoda A, Komatuzaki A, Muraoka H, Gou-Tsu K. A case with symptom of vestibular neuronitis caused by an intra-medullary lesion. J Laryngol Otol 112: 772-774, 1995.

6. Laigle-Donadey F, Taillibert S, Martin-Duverneuil N, Hildebrand J, Delattre JY. Skull-base metastases. J Neurooncol 75: 63-69, 2005.

7. Cherif El, Asri A, El Mostarchid B, Akhaddar A, Boucetta M. Chronic subdural hematoma revealing skull metastasis. Intern Med 50: 791, 2011.

8. Imre A, Sakarya EU, Imre SS, Gundogan O, Erdogan N, Rezanko T. Orbital apex syndrome as a sign of unsuspected breast carcinoma. J Craniofac Surg 24: 1476-1478, 2013.

9. Eckardt AM, Rana M, Essig H, Gellrich NC. Orbital metastases as first sign of metastatic spread in breast cancer: case report and review of the literature. Head Neck Oncol 3: 37, 2011.

10. Tomizawa Y, Ocque R, Ohori NP. Orbital metastasis as the initial presentation of invasive lobular carcinoma of breast. Intern Med 51: 1635-1638, 2012.

11. Takeuchi H, Tsuji K, Ueo H. Prediction of early and late recurrence in patients with breast carcinoma. Breast Cancer 12: 161$165,2005$.

12. Rotolo N, Dominioni L, De Monte L, Conti V, La Rosa S, Imperatori A. Metastasis at a tracheostomy site as the presenting sign of late recurrent breast cancer. Head Neck 35: E359-E362, 2013.

13. Chodorowski Z, Sein Anand J, Jassem J, et al. Late recurrence of malignant tumours 20 years after diagnosis and treatment. Przegl Lek 64: 372-373, 2007.

14. Voduc KD, Cheang MC, Tyldesley S, Gelmon K, Nielsen TO, Kennecke H. Breast cancer subtypes and the risk of local and regional relapse. J Clin Oncol 28: 1684-1691, 2010.

15. Ono M, Kosaka N, Tominaga N, et al. Exosomes from bone marrow mesenchymal stem cells contain a microRNA that promotes dormancy in metastatic breast cancer cells. Sci Signal 7: ra63, 2014.

(C) 2016 The Japanese Society of Internal Medicine http://www.naika.or.jp/imonline/index.html 